\title{
Stability of the Magnetic Monopole Condensate in three- and four-colour QCD
}

\author{
Michael L. Walker \\ Department of Physics, Faculty of Science, Chiba University, Chiba, 263-8522 Japan \\ E-mail: m.walker@aip.org.au
}

\begin{abstract}
It is argued that the ground state of three- and four-colour QCD contains a monopole condensate, necessary for the dual Meissner effect to be the mechanism of confinement, and support its stability on the grounds that it gives the off-diagonal gluons an effective mass sufficient to remove the unstable ground state mode.
\end{abstract}

KEYWORDS: QCD, confinement, magnetic condensate, magnetic monopoles. 


\section{Contents}

1. Introduction 1

2. Specifying Abelian Directions 2

3. The $S U(3)$ CFN QCD Vacuum

4. $U(1)^{N-1}$ Monopoles vs $U(N-1)$ Monopoles

5. Mass of Off-diagonal $S U(3)$ Gluons 5

6. Is the Monopole Condensate Stable in $S U(3)$ QCD? 6

7. Monopole vs the Magnetic Condensate 6

8. Stability of $S U(4)$ QCD 9

9. Discussion 9

\section{Introduction}

Proof of colour confinement is one of the most important, long-running problems in quantum field theory today. Thanks to the efforts of many authors, such as [1, 2, 3, 4, 5, 6], we may now be close to solving this puzzle. A particularly promising mechanism is the dual Meissner effect, in which a condensate of chromomagnetic monopoles excludes the chromoelectric field analogously to the Cooper pairs in a superconductor excluding the magnetic field. This proposal, dating back to the middle 1970s [7, 8, 9, 10, 11], requires QCD to have a magnetic monopole condensate. One obvious difficulty was ensuring that the magnetic condensate was due to monopoles, but the most discouraging was the result of Nielsen and Olesen [12] in two-colour QCD that a magnetic condensate renders the zero-point gluon fluctuations unstable. Although this instability was disputed 113, 14, 15, 16], its existence remained conventional wisdom until relatively recently. Cho et. al., using subtle causality considerations, have argued that Nielsen and Olesen's analysis was too näive and found instead that the imaginary part of the effective action was zero for magnetic backgrounds but non-zero for electric backgrounds [6]. Together with the current author, they have supported their result with independent calculations [17, 18], and recently extended it to three or more colours [19]. A different approach, taken by Kondo [20] in two-colour QCD, demonstrates the generation of an effective gluon mass large enough to remove the tachyon mode. We shall see that this argument has parallels with that of Flory [16] and Kay et.al. [21]. 
I shall repeat Kondo's approach in three- and four-colour QCD. Section 2 presents the Cho-Faddeev-Niemi (CFN) decomposition for general $S U(N)$ gauge groups. Section 3 determines the magnetic and monopole condensates, drawing heavily on the maximal abelian gauge analysis of Flyvbjerg [22]. This is followed by a discussion of the monopole generating subgroups $U(1)^{N-1}$ and $U(N-1)$ and the different roles they play confining gluons or quarks in section 4 . I study the gluon's effective mass matrix and determine the effective mass (squared) in terms of the magnitude of the monopole field in section 5 . The apparent instability is briefly discussed in section 6. I establish inequalities between the magnetic and monopole condensates in section 7. Section 8 adapts this approach to four-colour QCD.

\section{Specifying Abelian Directions}

The CFN decomposition was first presented by Cho [11], and later by Faddeev and Niemi [23], as a gauge-invariant means of specifying the Abelian dynamics of two-colour QCD. These authors [24, 25] also applied it to three-colour QCD. In this section we adapt it to general $S U(N)$, although we are not the first to do so [26, 27], and establish our notation.

The Lie group $S U(N)$ for $N$-colour QCD has $N^{2}-1$ generators $\lambda^{(i)}$, of which $N-1$ are Abelian generators $\Lambda^{(i)}$. For simplicity, we specify the gauge transformed Abelian

directions with $\hat{\mathbf{n}}_{i}=U^{\dagger} \Lambda^{(i)} U$. Fluctuations in the $\hat{\mathbf{n}}_{i}$ directions are described by $c_{\mu}^{(i)}$. The gauge field of the covariant derivative which leaves the $\hat{\mathbf{n}}_{i}$ invariant is given by

$$
g \mathbf{V}_{\mu} \times \hat{\mathbf{n}}_{i}=-\partial_{\mu} \hat{\mathbf{n}}_{i}
$$

In general this is

$$
\mathbf{V}_{\mu}=c_{\mu}^{(i)} \hat{\mathbf{n}}_{i}+\mathbf{B}_{\mu}, \mathbf{B}_{\mu}=g^{-1} \partial_{\mu} \hat{\mathbf{n}}_{i} \times \hat{\mathbf{n}}_{i},
$$

where summation is implied over $i$.

We define the covariant derivative

$$
\hat{\mathbf{D}}_{\mu}=\partial_{\mu}+g \mathbf{V}_{\mu} \times
$$

The monopole field strength

$$
\vec{H}_{\mu \nu}=\partial_{\mu} \mathbf{B}_{\nu}-\partial_{\nu} \mathbf{B}_{\mu}+g \mathbf{B}_{\mu} \times \mathbf{B}_{\nu}
$$

has only $\hat{\mathbf{n}}_{i}$ components, $i e$.

$$
H_{\mu \nu}^{(i)} \hat{\mathbf{n}}_{i}=\vec{H}_{\mu \nu},
$$

where $H_{\mu \nu}^{(i)}$ has the eigenvalue $H^{(i)}$. Since we are only concerned with magnetic backgrounds, $H^{(i)}$ is considered the magnitude of a background magnetic field $\mathbf{H}^{(i)}$.

$\mathbf{X}_{\mu}$ is defined to be the dynamical degrees of freedom (DOF) perpendicular to $\hat{\mathbf{n}}_{i}$, so if $\mathbf{A}_{\mu}$ is the gluon field then

$$
\mathbf{A}_{\mu}=\mathbf{V}_{\mu}+\mathbf{X}_{\mu}=c_{\mu}^{(i)} \hat{\mathbf{n}}_{i}+\mathbf{B}_{\mu}+\mathbf{X}_{\mu}
$$


where

$$
\mathbf{X}_{\mu} \perp \hat{\mathbf{n}}_{i}, \mathbf{X}_{\mu}=g^{-1} \hat{\mathbf{n}}_{i} \times \mathbf{D}_{\mu} \hat{\mathbf{n}}_{i}, \mathbf{D}_{\mu}=\partial_{\mu}+g \mathbf{A}_{\mu} \times
$$

Substituting the CFN decomposition into the QCD field strength tensor gives

$$
\begin{aligned}
\vec{F}^{2}= & \left(\partial_{\mu} c_{\nu}^{(i)}-\partial_{\nu} c_{\mu}^{(i)}\right)^{2}+\left(\partial_{\mu} \mathbf{B}_{\nu}-\partial_{\nu} \mathbf{B}_{\mu}+g \mathbf{B}_{\mu} \times \mathbf{B}_{\nu}\right)^{2} \\
& +2\left(\partial_{\mu} c_{\nu}^{(i)}-\partial_{\nu} c_{\mu}^{(i)}\right) \hat{\mathbf{n}}_{i} \cdot\left(\partial_{\mu} \mathbf{B}_{\nu}-\partial_{\nu} \mathbf{B}_{\mu}+g \mathbf{B}_{\mu} \times \mathbf{B}_{\nu}\right)+\left(\hat{\mathbf{D}}_{\mu} \mathbf{X}_{\nu}-\hat{\mathbf{D}}_{\nu} \mathbf{X}_{\mu}\right)^{2} \\
& +2 g\left(\left(\partial_{\mu} c_{\nu}^{(i)}-\partial_{\nu} c_{\mu}^{(i)}\right) \hat{\mathbf{n}}_{i}+\partial_{\mu} \mathbf{B}_{\nu}-\partial_{\nu} \mathbf{B}_{\mu}+g \mathbf{B}_{\mu} \times \mathbf{B}_{\nu}\right) \cdot\left(\mathbf{X}_{\mu} \times \mathbf{X}_{\nu}\right) \\
& +g^{2}\left(\mathbf{X}_{\mu} \times \mathbf{X}_{\nu}\right)^{2}+2 g\left(\hat{\mathbf{D}}_{\mu} \mathbf{X}_{\nu}-\hat{\mathbf{D}}_{\nu} \mathbf{X}_{\mu}\right) \cdot\left(\mathbf{X}_{\mu} \times \mathbf{X}_{\nu}\right) .
\end{aligned}
$$

This expression holds for all $N$-colour QCD except $N=2$ where the last term is absent.

A näive substitution of the CFN decomposition appears to leave the gluon field with additional DOF, and this has been a source of considerable confusion and controversy. Detailed analyses can be found in [28, 29, 30] demonstrating that the $\hat{\mathbf{n}}_{i}$ are not fundamental, but a compound of dynamic fields. Hence $\hat{\mathbf{n}}_{i}, \mathbf{B}_{\mu}$ are dynamic but do not constitute extra DOFs.

However the CFN decomposition does introduce additional gauge DOFs, which a proper application must fix. [6, 28] discussed the problem effectively in terms of the passive and active gauge symmetries, but I shall follow the notation of [29]. Their analysis was restricted to two-colour QCD, but its application to $N$-colours is so straightforward as to be little more than repetition. It is sufficient for our purposes to say that the CFN decomposition of QCD can be properly quantised in a consistent manner that leaves it equivalent to conventional QCD.

\section{The $S U(3)$ CFN QCD Vacuum}

To discuss the vacuum state we employ the formalism of Lie algebra roots to the isovectors $\mathbf{B}_{\mu}, \mathbf{X}_{\mu}$, reducing them to

$$
\begin{aligned}
& \mathbf{B}_{\mu}=\mathbf{B}_{\mu}^{(1,0)}+\mathbf{B}_{\mu}^{\left(\frac{1}{2}, \frac{\sqrt{3}}{2}\right)}+\mathbf{B}_{\mu}^{\left(\frac{1}{2},-\frac{\sqrt{3}}{2}\right)}=\mathbf{B}_{\mu}^{(1,0)}+\mathbf{B}_{\mu}^{\left(-\frac{1}{2},-\frac{\sqrt{3}}{2}\right)}+\mathbf{B}_{\mu}^{\left(-\frac{1}{2}, \frac{\sqrt{3}}{2}\right)} \\
& \mathbf{X}_{\mu}=\mathbf{X}_{\mu}^{(1,0)}+\mathbf{X}_{\mu}^{\left(\frac{1}{2}, \frac{\sqrt{3}}{2}\right)}+\mathbf{X}_{\mu}^{\left(\frac{1}{2},-\frac{\sqrt{3}}{2}\right)}=\mathbf{X}_{\mu}^{(1,0)}+\mathbf{X}_{\mu}^{\left(-\frac{1}{2},-\frac{\sqrt{3}}{2}\right)}+\mathbf{X}_{\mu}^{\left(-\frac{1}{2}, \frac{\sqrt{3}}{2}\right)}
\end{aligned}
$$

$\mathbf{B}_{\mu}^{(\alpha)}$ is defined so that

$$
g \mathbf{B}_{\mu}^{(\alpha)} \times \mathbf{B}_{\nu}^{(\alpha)}=-\vec{H}_{\mu \nu}^{(\alpha)}
$$

while $\mathbf{X}_{\mu}^{(\alpha)}$ is the component of $\mathbf{X}_{\mu}$ which feels the monopole field strength tensor $\vec{H}_{\mu \nu}^{(\alpha)}$, where

$$
\vec{H}_{\mu \nu}^{(\alpha)}=\alpha_{j} H_{\mu \nu}^{(j)}
$$

We also define the background magnetic field

$$
\mathbf{H}^{(\alpha)}=\alpha_{j} \mathbf{H}^{(j)},
$$


whose magnitude $H^{(\alpha)}$ is $\vec{H}_{\mu \nu}^{(\alpha)}$ 's non-zero eigenvalue. It follows that

$$
H^{(1,0)}=H^{(1)}, \quad H^{\left(\frac{1}{2}, \pm \frac{\sqrt{3}}{2}\right)^{2}}=\frac{1}{4} H^{(1)^{2}}+\frac{3}{4} H^{(2)^{2}} \pm \frac{\sqrt{3}}{2} \mathbf{H}^{(1)} \cdot \mathbf{H}^{(2)} .
$$

This result is formally the same as Flyvberg's [22], with the subtle difference that our $H^{(\alpha)}$ refers to the the field strength generated by the Cho connection while Flyvberg's is simply the field strength along the Abelian directions in the maximal Abelian gauge. Nonetheless, it is clear that we can repeat the renormalization analysis and get the same formal result. This gives the corresponding results for the lowest energy state,

$$
H^{(1)}=H^{(2)}, \mathbf{H}^{(1)} \perp \mathbf{H}^{(2)},
$$

as found independently for the CFN formalism using a different approach by Cho, Kim and Pak [19].

\section{4. $U(1)^{N-1}$ Monopoles vs $U(N-1)$ Monopoles}

Since the ultimate motivation of this work is confinement, it is appropriate to discuss an important issue first brought to light by Kondo and Taira [31, 32] in their construction of a non-Abelian version of Stokes' theorem. They found that the monopole contribution to the Wilson loop depends on which representation of the gauge group the colour charge belongs to. In the $S U(3)$ gauge group for example, discussion of the fundamental representation concerns only the monopoles corresponding to the reduction from $S U(3)$ down to $U(2)$ symmetry [31], specified by the homotopy group

$$
\pi_{2}[S U(3) / U(2)]=\pi_{1}[U(2)]=\pi_{1}\left[S U(2) \otimes U_{2}(1)\right]=\pi_{1}\left[U_{2}(1)\right]=\mathbb{Z}_{2},
$$

(Subscripts ${ }_{i}$ in this section denote the relevant Abelian generator $\Lambda^{(i)}$.) while for colour charges in the adjoint representation we need to consider the corresponding $U(1) \otimes U(1)$ fundamental group

$$
\pi_{2}\left[S U(3) /\left(U_{1}(1) \otimes U_{2}(1)\right)\right]=\pi_{1}\left[U_{1}(1) \otimes U_{2}(1)\right]=\mathbb{Z}_{1} \oplus \mathbb{Z}_{2} .
$$

The Abelian generator $\Lambda^{(1)}$ of the subgroup $U(2)$ is contained in the simply connected subgroup $S U(2)$, leaving only the fundamental group generated by $\Lambda^{(2)}$. Hence the monopole charges corresponding to the $U(2)$ subgroup are a subset of those corresponding to $U_{1}(1) \otimes$ $U_{2}(1)$. Specifically, it is the subset for which the charge corresponding to $U_{1}(1)$ is zero.

To construct the monopole field due to the $U(2)$ subgroup, observe that since the $U(2)$ monopole field is purely $\Lambda^{(2)}$-like it will be the covariant connection of the unit vector $\hat{\mathbf{n}}_{2}$. It is easy to show that

$$
\mathbf{L}_{\mu}=g^{-1} \frac{4}{3} \partial_{\mu} \hat{\mathbf{n}}_{2} \times \hat{\mathbf{n}}_{2}
$$

has the required property

$$
g \mathbf{L}_{\mu} \times \hat{\mathbf{n}}_{2}=-\partial_{\mu} \hat{\mathbf{n}}_{2}
$$


Of course, the $U_{1}(1) \otimes U_{2}(1)$ monopole field $\mathbf{B}_{\mu}$ also has this property, so

$$
g\left(\mathbf{B}_{\mu}-\mathbf{L}_{\mu}\right) \times \hat{\mathbf{n}}_{2}=0 .
$$

Since

$$
g \mathbf{B}_{\mu}^{(1,0)} \times \hat{\mathbf{n}}_{2}=0
$$

it follows that

$$
\mathbf{L}_{\mu}=\mathbf{B}_{\mu}^{\left(\frac{1}{2}, \frac{\sqrt{3}}{2}\right)}+\mathbf{B}_{\mu}^{\left(\frac{1}{2},-\frac{\sqrt{3}}{2}\right)}=\mathbf{B}_{\mu}^{\left(-\frac{1}{2},-\frac{\sqrt{3}}{2}\right)}+\mathbf{B}_{\mu}^{\left(-\frac{1}{2}, \frac{\sqrt{3}}{2}\right)}
$$

For general $S U(N)$, the Wilson loop for gluons, which belong to the adjoint representation, depends on the full set of monopoles corresponding to the homotopy group

$$
\pi_{2}\left[S U(N) /\left(U_{1}(1)^{N-1}\right)\right]=\pi_{1}\left[U_{1}(1)^{N-1}\right]=\mathbb{Z}_{1} \oplus \mathbb{Z}_{2} \oplus \ldots \oplus \mathbb{Z}_{N-1}
$$

This is in contrast to that of quarks in the fundamental representation, which receives monopole contributions only from those corresponding to

$$
\pi_{2}[S U(N) / U(N-1)]=\pi_{1}[U(N-1)]=\pi_{1}\left[U_{N-1}(1)\right]=\mathbb{Z}_{N-1} .
$$

The covariant connection of the unit vector $\hat{\mathbf{n}}_{N-1}$ has the general form

$$
\mathbf{L}_{\mu}=g^{-1} K(N) \partial_{\mu} \hat{\mathbf{n}}_{N-1} \times \hat{\mathbf{n}}_{N-1} .
$$

It is now trivial to generalise the $S U(3)$ statement

$$
\left(\mathbf{B}_{\mu}-\mathbf{L}_{\mu}\right) \times \hat{\mathbf{n}}_{N-1}=0
$$

This section demonstrates that a whole new analysis is unnecessary if quark, rather than gluon confinement is of interest. Of course, stability of the ground-state fluctuations of the quark field was never an issue. In the expected absence of internal anisotropy, all other results concerning condensates of $\mathbf{B}_{\mu}$ should also hold for $\mathbf{L}_{\mu}$.

\section{Mass of Off-diagonal $S U(3)$ Gluons}

Following Kondo [20], we observe at the classical level that the monopole condensate gives the off-diagonal gluons an effective mass via

$$
\frac{1}{2}\left(\hat{\mathbf{D}}_{\mu} \mathbf{X}_{\nu}-\hat{\mathbf{D}}_{\nu} \mathbf{X}_{\mu}\right)^{2} \stackrel{I B P}{\longrightarrow}\left(\mathbf{X}_{\mu} \hat{\mathbf{D}}_{\nu}\right) \cdot\left(\hat{\mathbf{D}}_{\mu} \mathbf{X}_{\nu}\right)-\left(\mathbf{X}_{\mu} \hat{\mathbf{D}}_{\nu}\right) \cdot\left(\hat{\mathbf{D}}_{\nu} \mathbf{X}_{\mu}\right)
$$

The latter term gives

$$
g^{2} B_{\rho}^{D} X_{\mu}^{E} B_{\rho}^{B} X_{\mu}^{C} f_{A B C} f_{A D E}
$$

which provides the effective gluon mass matrix

$$
M_{E C}^{2}=g^{2} B_{\rho}^{D} B_{\rho}^{B} f_{A B C} f_{A D E}
$$


Since the effective mass term arises from the quartic gluon terms, this is consistent with an early calculation by Flory [16] and Kay et.al. [21] showing that the instability is removed when the quartic terms relevant to the unstable modes are included. Dudal and coworkers are following an entirely different approach [33, 34] in which the gluon mass comes from a ghost-gluon condensate with dimensions of mass squared.

So far this section has followed the corresponding section 2.2 in [20]. Because the algebra of $S U(2)$ is simpler than that of $S U(3)$, the author was able to simply diagonalize the mass matrix and obtain the mass squared eigenvalues $\mathbf{B} \cdot \mathbf{B}$ (multiplicity two) and zero. The zero eigenvalue corresponds to the Abelian direction.

Diagonalizing (5.3) however, is too difficult even for mathematica but there is another way. The sum of the mass eigenvalues is the trace of the mass matrix, $3 g^{2} \mathbf{B} \cdot \mathbf{B}$. Since there are two Abelian directions from which the valence gluon is excluded by definition (see (2.7)), it follows that zero is an eigenvalue of multiplicity two and the average effective mass squared is

$$
M_{X}^{2}=\frac{3}{8-2} g^{2} \mathbf{B} \cdot \mathbf{B}=\frac{1}{2} g^{2} \mathbf{B} \cdot \mathbf{B} .
$$

Since all physical masses are equal by the isotropy of the condensate and the gauge invariance of the mass term (5.2), (5.4) is the effective mass of all valence gluons. A conventional diagonalization of $M_{E C}^{2}$ in this treatment would, of course have been preferable, but this approach does give the same result as diagonalization in $S U(2)$ QCD.

\section{Is the Monopole Condensate Stable in $S U(3)$ QCD?}

It has been shown 22, 35] that

$$
\left\|\mathbf{H}^{(\alpha)}\right\| \neq 0
$$

but a calculation of the $\mathbf{X}^{(\alpha)}$ ground-state energy using zeta-function renormalization, as first demonstrated in two-colour QCD [12] by Nielsen and Olesen, has an imaginary contribution [36] from

$$
\sqrt{\mathbf{k}^{2}-g\left\|\mathbf{H}^{(\alpha)}\right\|}
$$

However there is still hope, because we saw in section 5 that the gluons gain an effective mass, changing this to

$$
\sqrt{\mathbf{k}^{2}+M_{X}^{2}-g\left\|\mathbf{H}^{(\alpha)}\right\|}
$$

It now remains to demonstate that the spin contribution is smaller in magnitude than the effective gluon mass squared.

\section{Monopole vs the Magnetic Condensate}

Since $\left\langle\left\|H^{(\alpha)}\right\|\right\rangle$ does not vary with $\alpha$, proving sufficient $M_{X}^{2}$ to prevent tachyons for $\mathbf{X}_{\mu}^{(1,0)}$ is sufficient to prove it for $\mathbf{X}_{\mu}$. Noting

$$
\left(\mathbf{B}_{\mu}^{(\alpha)} \times \mathbf{B}_{\nu}^{(\alpha)}\right)^{2}=\left(\mathbf{B}_{\mu}^{(\alpha)} \cdot \mathbf{B}_{\mu}^{(\alpha)}\right)^{2}-\left(\mathbf{B}_{\mu}^{(\alpha)} \cdot \mathbf{B}_{\nu}^{(\alpha)}\right)^{2} .
$$


gives

$$
\begin{aligned}
& \left\|g \mathbf{H}^{(1,0)}\right\| \\
& =g^{2}\left\|\hat{\mathbf{n}}_{1} \cdot \mathbf{B}_{\mu}^{(1,0)} \times \mathbf{B}_{\nu}^{(1,0)}+\hat{\mathbf{n}}_{1} \cdot \mathbf{B}_{\mu}^{\left(\frac{1}{2}, \frac{\sqrt{3}}{2}\right)} \times \mathbf{B}_{\nu}^{\left(\frac{1}{2}, \frac{\sqrt{3}}{2}\right)}+\hat{\mathbf{n}}_{1} \cdot \mathbf{B}_{\mu}^{\left(\frac{1}{2},-\frac{\sqrt{3}}{2}\right)} \times \mathbf{B}_{\nu}^{\left(\frac{1}{2},-\frac{\sqrt{3}}{2}\right)}\right\| \\
& \leq \frac{2 g^{2}}{3} \mathbf{B} \cdot \mathbf{B}
\end{aligned}
$$

which is not strong enough. We remedy this by showing that

$$
\sqrt{2}\left\|\mathbf{B}_{\mu}^{(\alpha)} \times \mathbf{B}_{\nu}^{(\alpha)}\right\| \leq \mathbf{B}_{\mu}^{(\alpha)} \cdot \mathbf{B}_{\mu}^{(\alpha)}
$$

Begin by constructing a convenient coordinate system. Let $\left\{\overline{\mathbf{n}}_{i}\right\}_{i=1}^{N^{2}-1}$ be unit vectors spanning $S U(N) \cdot \overline{\mathbf{n}}_{i} \cdot \overline{\mathbf{n}}_{j} \times \overline{\mathbf{n}}_{k}$ is gauge invariant under

$$
\delta \overline{\mathbf{n}}_{i}=\overline{\mathbf{n}}_{i} \times \alpha,
$$

so

$$
\overline{\mathbf{n}}_{i} \cdot \overline{\mathbf{n}}_{j} \times \overline{\mathbf{n}}_{k} \equiv \bar{f}_{i j k}=f_{i j k} .
$$

We can construct a convenient coordinate system by starting with

$$
\left\{\overline{\mathbf{n}}_{i}\right\}=\left\{\hat{\mathbf{e}}_{i}\right\},
$$

and gauge transforming $\left\{\overline{\mathbf{n}}_{i}\right\}$ so that

$$
\overline{\mathbf{n}}_{3}, \overline{\mathbf{n}}_{8}=\hat{\mathbf{n}}_{1}, \hat{\mathbf{n}}_{2}
$$

respectively. It follows that

$$
\bar{f}_{i j k}=f_{i j k} .
$$

Restricting the analysis to $S U(3), \mathbf{B}_{\mu}^{ \pm(1,0)}, \mathbf{X}_{\mu}^{ \pm(1,0)}$ lie in the $\left\{\overline{\mathbf{n}}_{1}, \overline{\mathbf{n}}_{2}\right\}$ plane, $\mathbf{B}_{\mu}^{ \pm\left(\frac{1}{2}, \frac{\sqrt{3}}{2}\right)}, \mathbf{X}_{\mu}^{ \pm\left(\frac{1}{2}, \frac{\sqrt{3}}{2}\right)}$ lie in the $\left\{\overline{\mathbf{n}}_{4}, \overline{\mathbf{n}}_{5}\right\}$ plane, and $\mathbf{B}_{\mu}^{ \pm\left(\frac{1}{2},-\frac{\sqrt{3}}{2}\right)}, \mathbf{X}_{\mu}^{ \pm\left(\frac{1}{2},-\frac{\sqrt{3}}{2}\right)}$ lie in the $\left\{\overline{\mathbf{n}}_{6}, \overline{\mathbf{n}}_{7}\right\}$ plane.

The following is based on a method developed by Kondo [37] for two-colour QCD. Define

$$
T^{a b}=\bar{n}^{a} \cdot \partial \hat{\mathbf{n}}_{1} \bar{n}^{b} \cdot \partial \hat{\mathbf{n}}_{1}
$$

where $a, b$ are restricted to $1,2 . T^{a b}$ is a two by two matrix, having two real eigenvalues, $\lambda_{1}$ and $\lambda_{2}$ say. We find the inequality

$$
\frac{1}{2}\left(\sum_{a=1}^{2} \lambda_{a}\right)^{2}=\lambda_{1}^{2}+\lambda_{2}^{2}-\frac{1}{2}\left(\lambda_{1}-\lambda_{2}\right)^{2} \leq \sum_{a=1}^{2} \lambda_{a}^{2} \rightarrow \frac{1}{2}(\operatorname{Tr} T)^{2} \leq\left(\operatorname{Tr} T^{2}\right) .
$$

Proof of $(7.3)$ for $H_{\mu \nu}^{(1,0)}$ is straightforward. Take

$$
\left[\partial_{\mu} \hat{\mathbf{n}}_{1}\right]^{a}=\bar{n}^{a} \bar{n}^{a} \cdot \partial_{\mu} \hat{\mathbf{n}}_{1} \text { (no summation), }
$$


where $a$ is restricted to 1,2 . We get

$$
\begin{aligned}
g^{2} \vec{H}_{\mu \nu}^{(1,0)} \cdot \vec{H}_{\mu \nu}^{(1,0)} & =g^{4}\left(\mathbf{B}_{\mu}^{(1,0)} \times \mathbf{B}_{\nu}^{(1,0)}\right)^{2}=\bar{f}^{3 a b}\left[\partial_{\mu} \hat{\mathbf{n}}_{1}\right]^{a}\left[\partial_{\nu} \hat{\mathbf{n}}_{1}\right]^{b} \bar{f}^{3 c d}\left[\partial_{\mu} \hat{\mathbf{n}}_{1}\right]^{c}\left[\partial_{\nu} \hat{\mathbf{n}}_{1}\right]^{d} \\
& =(\operatorname{Tr} T)^{2}-\left(\operatorname{Tr} T^{2}\right) .
\end{aligned}
$$

Substituting in $(7.9)$ we find

$$
\vec{H}_{\mu \nu}^{(1,0)} \cdot \vec{H}_{\mu \nu}^{(1,0)} \leq \frac{1}{2} g^{2}\left(\mathbf{B}_{\mu}^{(1,0)} \cdot \mathbf{B}_{\mu}^{(1,0)}\right)^{2},
$$

which leads to equation (7.3). The construction for $\vec{H}^{ \pm\left(\frac{1}{2}, \frac{\sqrt{3}}{2}\right)}$ is only slightly more complicated. Redefine

$$
T^{(a-3)(b-3)}=\bar{n}^{a} \cdot \partial \hat{\mathbf{n}}_{2} \bar{n}^{b} \cdot \partial \hat{\mathbf{n}}_{2}
$$

where $a, b$ are restricted to 4,5 . Now take

$$
\left[\partial_{\mu} \hat{\mathbf{n}}_{2}\right]^{a}=\bar{n}^{a} \bar{n}^{a} \cdot \partial_{\mu} \hat{\mathbf{n}}_{2}
$$

where $a$ is still restricted to 4,5. Recalling the discussion of equation (4.3) and repeating the above argument leads to

$$
\begin{aligned}
g^{2} & \vec{H}_{\mu \nu}^{ \pm\left(\frac{1}{2}, \frac{\sqrt{3}}{2}\right)} \cdot \vec{H}_{\mu \nu}^{ \pm\left(\frac{1}{2}, \frac{\sqrt{3}}{2}\right)}=g^{4}\left(\mathbf{B}_{\mu}^{ \pm\left(\frac{1}{2}, \frac{\sqrt{3}}{2}\right)} \times \mathbf{B}_{\nu}^{ \pm\left(\frac{1}{2}, \frac{\sqrt{3}}{2}\right)}\right)^{2} \\
& =\frac{16}{9}\left(\frac{1}{4} \bar{f}^{3 a b} \bar{f}^{3 c d}+\frac{3}{4} \bar{f}^{8 a b} \bar{f}^{8 c d}\right)\left[\partial_{\mu} \hat{\mathbf{n}}_{2}\right]^{a}\left[\partial_{\nu} \hat{\mathbf{n}}_{2}\right]^{b}\left[\partial_{\mu} \hat{\mathbf{n}}_{2}\right]^{c}\left[\partial_{\nu} \hat{\mathbf{n}}_{2}\right]^{d} \\
& =\frac{16}{9}\left((\operatorname{Tr} T)^{2}-\left(\operatorname{Tr} T^{2}\right)\right),
\end{aligned}
$$

which again yields equation $(7.3)$. The argument for $\vec{H}^{ \pm\left(\frac{1}{2}, \frac{\sqrt{3}}{2}\right)}$ is identical. The adaptation of this technique to higher $N$ is straightforward.

The above introduces a factor of $\sqrt{2}$ to the inequality $(7.2)$, which becomes

$$
\begin{aligned}
& \left\|g \mathbf{H}^{(1,0)}\right\| \\
& =g^{2}\left\|\hat{\mathbf{n}}_{1} \cdot \mathbf{B}_{\mu}^{(1,0)} \times \mathbf{B}_{\nu}^{(1,0)}+\hat{\mathbf{n}}_{1} \cdot \mathbf{B}_{\mu}^{\left(\frac{1}{2}, \frac{\sqrt{3}}{2}\right)} \times \mathbf{B}_{\nu}^{\left(\frac{1}{2}, \frac{\sqrt{3}}{2}\right)}+\hat{\mathbf{n}}_{1} \cdot \mathbf{B}_{\mu}^{\left(\frac{1}{2},-\frac{\sqrt{3}}{2}\right)} \times \mathbf{B}_{\nu}^{\left(\frac{1}{2},-\frac{\sqrt{3}}{2}\right)}\right\| \\
& \leq \frac{2 g^{2}}{3 \sqrt{2}} \mathbf{B} \cdot \mathbf{B}<\frac{1}{2} g^{2} \mathbf{B} \cdot \mathbf{B},
\end{aligned}
$$

demonstrating that the effective mass is sufficient to stabilize the tachyonic gluon mode.

While unnecessary for $S U(3)$, it is possible to use $\left\langle\left\|H^{(1)}\right\|\right\rangle=\left\langle\left\|H^{(2)}\right\|\right\rangle$ to find an even stronger upper bound on $\left\langle\left\|\vec{H}^{(\alpha)}\right\|\right\rangle$.

$$
\left\|g \mathbf{H}^{(2)}\right\| \leq g^{2} \frac{\sqrt{3}}{2}\left(\left\|\mathbf{B}_{\mu}^{\left(\frac{1}{2}, \frac{\sqrt{3}}{2}\right)} \times \mathbf{B}_{\nu}^{\left(\frac{1}{2}, \frac{\sqrt{3}}{2}\right)}+\mathbf{B}_{\mu}^{\left(\frac{1}{2},-\frac{\sqrt{3}}{2}\right)} \times \mathbf{B}_{\nu}^{\left(\frac{1}{2},-\frac{\sqrt{3}}{2}\right)}\right\|\right),
$$

yielding

$$
\left\|g \mathbf{H}^{(2)}\right\| \leq g^{2} \frac{\sqrt{3}}{3 \sqrt{2}} \mathbf{B} \cdot \mathbf{B}=\frac{g^{2}}{\sqrt{6}} \mathbf{B} \cdot \mathbf{B}<\frac{g^{2} \sqrt{2}}{3} \mathbf{B} \cdot \mathbf{B} .
$$

This style of argument will prove necessary in the treatment of $S U(4)$. 


\section{Stability of $S U(4)$ QCD}

Repeating the analysis of section 5 finds an effective gluon mass squared in $S U(4)$ QCD of

$$
M_{S U(4)}^{2}=\frac{4 g^{2} \mathbf{B} \cdot \mathbf{B}}{12}=\frac{g^{2}}{3} \mathbf{B} \cdot \mathbf{B} .
$$

Following the last section, we again need only one $\left\langle\left\|g \mathbf{H}^{(i)}\right\|\right\rangle<M_{S U(4)}^{2}$. For $\mathbf{H}^{(1)}$ we get

$$
\left\|g \mathbf{H}^{(1)}\right\| \leq \frac{g^{2}}{2 \sqrt{2}} \mathbf{B} \cdot \mathbf{B}>M_{S U(4)}^{2}
$$

but studying $\mathbf{H}^{(3)}$ yields

$$
\begin{aligned}
\left\|g \mathbf{H}^{(3)}\right\| \leq & \sqrt{\frac{2}{3}} \| g^{2} \mathbf{B}_{\mu}^{\left(\frac{1}{2}, \sqrt{\frac{1}{12}}, \sqrt{\frac{2}{3}}\right)} \times \mathbf{B}_{\nu}^{\left(\frac{1}{2}, \sqrt{\frac{1}{12}}, \sqrt{\frac{2}{3}}\right) \|} \\
& +\sqrt{\frac{2}{3}}\left\|g^{2} \mathbf{B}_{\mu}^{\left(\frac{1}{2},-\sqrt{\frac{1}{12}},-\sqrt{\frac{2}{3}}\right)} \times \mathbf{B}_{\nu}^{\left(\frac{1}{2},-\sqrt{\frac{1}{12}},-\sqrt{\frac{2}{3}}\right)}\right\| \\
& +\sqrt{\frac{2}{3}}\left\|g^{2} \mathbf{B}_{\mu}^{\left(0, \sqrt{\frac{1}{3}},-\sqrt{\frac{2}{3}}\right)} \times \mathbf{B}_{\nu}^{\left(0, \sqrt{\frac{1}{3}},-\sqrt{\frac{2}{3}}\right)}\right\| \\
\leq & \frac{g^{2}}{2 \sqrt{3}} \mathbf{B} \cdot \mathbf{B}<M_{S U(4)}^{2},
\end{aligned}
$$

protecting the monopole condensate in $S U(4)$ QCD. It is pointless to try and generalize this result to arbitrary $S U(N>4)$ in three dimensional space because satisfying the equations (3.6) requires $N-1$ mutually orthogonal vector fields.

\section{Discussion}

A case for a stable monopole condensate in the QCD vacuum has been presented. By adapting the CFN decomposition to the higher gauge group we have ensured that our analysis describes the monopoles in a consistent, gauge invariant manner. Applying the CFN decomposition to $S U(N>2)$ is straightforward and reasonably intuitive. This was also the experience of Cho, Kim and Pak [19] who have demonstrated condensate stability in $S U(3)$ QCD by calculating the imaginary part of the effective action as discussed earlier.

The CFN formalism, while different from and superior to t'Hooft's Abelian gauge, has sufficient formal similarity for Flyvbjerg's analysis [22] to carry over to it, so we inherit the corresponding results concerning the QCD ground state in section 3 .

When discussing whether the effective gluon mass is sufficient to stabilize the ground state, it is important to remember that the relevant magnetic field strength magnitudes are found in the gluon spin interaction $\left\|\mathbf{H}^{(\alpha)}\right\|$. Diagonalizing the mass matrix directly seems impossible, but the invariance of the mass-generating term under global active gauge transformations ensures that the mass eigenvalues are equal, allowing their deduction from the trace of the mass matrix. It must be remembered that the construction of the gluon mass squared matrix was a classical one, even though a one-loop calculation is providing 
the non-zero condensate. Complete proof requires the mass matrix calculation to be a quantum one. The approach of Dudal and coworkers [33, 34] is interesting in this regard.

It has been shown explicitly for three-colour QCD that the CFN decomposition corresponding to the maximal Abelian subgroup contains the monopoles corresponding to the $U(2)$ subgroup automatically. It is not hard to derive the corresponding result for the CFN decomposition in four-colour QCD from the non-trivial homotopy groups for $S U(4)$ [38].

Our main result is that applying Kondo's argument [20] to $S U(3)$ or $S U(4)$ QCD finds an effective gluon mass sufficient to stabilize the monopole condensate. $S U(N>4)$ QCD requires a new analysis for reasons given at the end of section 8 .

\section{Acknowledgments}

The author wishes to thank K.-I. Kondo for many helpful discussions and for proof-reading the manuscript. He also thanks T. Shinohara and T. Murakami for many helpful discussions and criticisms. The author is supported by a postdoctoral fellowship from the Japan Society for the Promotion of Science (JSPS)

\section{References}

[1] Z. F. Ezawa and A. Iwazaki, Abelian dominance and quark confinement in yang-mills theories, Phys. Rev. D 25 (1982) 2681.

[2] T. Suzuki, A ginzburg-landau type theory of quark confinement, Prog. Theor. Phys. 80 (1988) 929.

[3] H. Suganuma, S. Sasaki, and H. Toki, Color confinement, quark pair creation and dynamical chiral symmetry breaking in the dual ginzburg-landau theory, Nucl. Phys. B 435 (1995) 207-240 hep-ph/9312350.

[4] K.-I. Kondo, Abelian-projected effective gauge theory of QCD with asymptotic freedom and quark confinement, Phys. Rev. D 57 (1998) 7467 hep-th/9709109.

[5] L. Freyhult, Field decomposition and the ground state structure of su(2) yang-mills theory, Int. J. Mod. Phys. A 17 (2002) 3681 hep-th/0106239.

[6] Y. M. Cho and D. G. Pak, Monopole condensation in su(2) qcd, Phys. Rev. D 65 (2002) 074027 hep-th/0201179.

[7] Y. Nambu, Strings, monopoles, and gauge fields, Phys. Rev. D 10 (1974) 4269.

[8] S. Mandelstam, Vortices and quark confinement in nonabelian gauge theories, Phys. Rept. 23 (1976) 245-249.

[9] A. M. Polyakov, Quark confinement and topology of gauge groups, Nucl. Phys. B 120 (1977) 429-458.

[10] G. 't Hooft, Topology of the gauge condition and new confinement phases in nonabelian gauge theories, Nucl. Phys. B 190 (1981) 455.

[11] Y. M. Cho, A restricted gauge theory, Phys. Rev. D 21 (1980) 1080.

[12] N. K. Nielsen and P. Olesen, An unstable yang-mills field mode, Nucl. Phys. B 144 (1978) 37 . 
[13] J. Honerkamp, The question of invariant renormalizability of the massless yang-mills theory in a manifest covariant approach, Nucl. Phys. B 48 (1972) 269.

[14] V. Schanbacher, Gluon propagator and effective lagrangian in qcd, Phys. Rev. D 26 (1982 48 .

[15] W. Dittrich and M. Reuter, Effective qcd lagrangian with zeta function regularization, Phys. Lett. B 128 (1983) 321.

[16] C. A. Flory, Covariant constant chromomagnetic fields and elimination of the one loop instabilities, . SLAC-PUB-3244.

[17] Y. M. Cho, M. L. Walker, and D. G. Pak, Monopole condensation and confinement of color in su(2) qcd, J. High Energy Phys. 05 (2004) 079 hep-th/0209208.

[18] Y. M. Cho and M. L. Walker, Stability of monopole condensation in su(2) qcd, Mod. Phys. Lett. A 19 (2004) 2707.

[19] Y. M. Cho, J. H. Kim, and D. G. Pak, Effective potential of qcd, hep-th/0511124.

[20] K.-I. Kondo, Magnetic condensation, abelian dominance and instability of savvidy vacuum, Phys. Lett. B 600 (2004) 287 hep-th/0404252].

[21] D. Kay, A. Kumar, and R. Parthasarathy, Savvidy vacuum in su(2) yang-mills theory, Mod. Phys. Lett. A 20 (2005) 1655.

[22] H. Flyvbjerg, Improved qcd vacuum for gauge groups su(3) and su(4), Nucl. Phys. B 176 (1980) 379 .

[23] L. D. Faddeev and A. J. Niemi, Partially dual variables in su(2) yang-mills theory, Phys. Rev. Lett. 82 (1999) 1624 hep-th/9807069.

[24] Y. M. Cho, Colored monopoles, Phys. Rev. Lett. 44 (1980) 1115.

[25] L. D. Faddeev and A. J. Niemi, Decomposing the yang-mills field, Phys. Lett. B 464 (1999 9 hep-th/9907180.

[26] L. D. Faddeev and A. J. Niemi, Partial duality in su(n) yang-mills theory, Phys. Lett. B 449 (1999) 21 hep-th/9812090.

[27] S. Li, Y. Zhang, and Z.-y. Zhu, Decomposition of su(n) connection and effective theory of su(n) qcd, Phys. Lett. B 487 (2000) 201 hep-th/9911132.

[28] W. S. Bae, Y. M. Cho, and S. W. Kimm, Qcd versus skyrme-faddeev theory, Phys. Rev. D 65 (2002) 025005 hep-th/0105163.

[29] K. I. Kondo, T. Murakami, and T. Shinohara, Brst symmetry of su(2) yang-mills theory in cho-faddeev- niemi decomposition, Eur. Phys. J. C 42 (2005) 47 hep-th/0504198.

[30] S. Kato et. al., Lattice construction of cho-faddeev-niemi decomposition and gauge invariant monopole, Phys. Lett. B 632 (2006) 326-339 hep-lat/0509069.

[31] K. Kondo and Y. Taira, Non-abelian stokes theorem and quark confinement in su(3) yang-mills gauge theory, Mod. Phys. Lett. A 15 (2000) 367.

[32] K.-I. Kondo and Y. Taira, Non-abelian stokes theorem and quark confinement in su(n) yang-mills gauge theory, Prog. Theor. Phys. 104 (2000) 1189 hep-th/9911242.

[33] D. Dudal et. al., An analytic study of the off-diagonal mass generation for yang-mills theories in the maximal abelian gauge, Phys. Rev. D 70 (2004) 114038 [hep-th/0406132]. 
[34] D. Dudal, H. Verschelde, R. E. Browne, and J. A. Gracey, The 2ppi expansion: Dynamical mass generation and vacuum energy, hep-th/0309241.

[35] G. K. Savvidy, Infrared instability of the vacuum state of gauge theories and asymptotic freedom, Phys. Lett. B 71 (1977) 139.

[36] M. L. Walker, in preparation.

[37] K.-I. Kondo, private communication.

[38] Y. D. Kim, I. G. Koh, and Y. J. Park, Complete set of su(4) monopole solutions, Phys. Rev. D 25 (1982) 58\%. 